\title{
Impact of Triaging for COVID-19 Patients in a Tertiary Care Hospital in West India: A Retrospective Analysis of a Case Series
}

\author{
Srishti S Jain ${ }^{1}$, Ashish Jain ${ }^{2}$, Anchin Kalia ${ }^{3}$, Pallaavi Goel ${ }^{4}$, Ravi Jain ${ }^{5}$, Nimish Mathur ${ }^{6}$, Puneet Rijhwani ${ }^{7}$, Anand Jain $^{8}$, \\ Divyansh Gupta ${ }^{9}$
}

\begin{abstract}
Introduction: Assessing the clinical severity of coronavirus disease-2019 (COVID-19) and triaging to appropriate levels of care is certainly one of the key elements in the success of managing COVID-19 patients. During the concluded wave of the pandemic, cases were categorized and cared for with set criteria prescribed by authorities. Other triaging criteria were included in contemporary international guidelines, but this hypothesis was never tested if anyone set is ever better than the other.

Materials and methods: This is a case series of 165 deceased patients of COVID-19. All patients were categorized as per clinical disease severity and admitted to the designated care area after confirmation of SARS-CoV-2 infection as decided by the admitting doctors. We collected retrospective data from patient medical records and analyzed for medical history, comorbidity profiles, hematology investigations, organ function tests, computed tomography of the thorax, and point-of-care biomarker test (D-dimer, procalcitonin, NT-proBNP, Trop-T). These data were analyzed to compare the differences between the variables of ward and ICU patients by using XLstat software.

Results: In this analysis of deceased patients' case series, we found that there was no significant difference among the patients admitted to ward and ICU for initial demographic and biomarker variables and risk factors. Diabetes was the most commonly found comorbidity. The mortality rate among the ward and ICU (5.89 vs $6.67 \%, p$ value: 0.44 ) was also similar among both the cohorts.

Conclusion: In this case series, we could conclude that both the cohorts were comparable at admission on demographic and laboratory parameter profile.

Clinical significance: This analysis led us to the conclusion that our existing "triage criteria" for COVID-19 patients will need appropriate modification before the second wave sets in the region.

Keywords: Biomarkers, Capacity management, COVID-19, Point-of-care testing, Radiological imaging, Triaging.

Journal of Mahatma Gandhi University of Medical Sciences \& Technology (2021): 10.5005/jp-journals-10057-0145
\end{abstract}

\section{INTRODUCTION}

The pandemic of coronavirus disease-2019 (COVID-19) has led to $112,209,815$ confirmed cases of COVID-19, including 2,490,776 deaths, reported to WHO. ${ }^{1}$ COVID-19 is truly a novel disease that caught the medical fraternity off guard in many ways, and to date, many new facts are revealed on daily basis. Assessing the clinical severity of COVID-19 and triaging to the appropriate level of care is now an established practice for the care of the patients and certainly one of the key elements in the success of managing COVID-19 patients. About $26-32 \%$ of all hospitalized patients were admitted to ICU, and mortality in ICU ranged from 39 to $72 \%$ according to initial reports. ${ }^{2-6}$ During the concluded wave of the pandemic, cases were categorized and cared for with these set criteria. ${ }^{7}$ Other triaging criteria were included in contemporary international guidelines, but this hypothesis was never tested if anyone set or any combination is ever better than the other. ${ }^{8}$

Retrospective observations have revealed that levels of biomarkers such as CRP, D-dimer, etc., may prove to be reliable indicators of disease severity and mortality. ${ }^{9-11}$

Now with the advent of point-of-care testing for biomarkers, these can be included in better triaging of COVID-19 patients.

Presently mankind is engaged with many emerging strains of SARS-CoV-2 and our country is also considered to be on the verge of an imminent follow-up wave of this pandemic. ${ }^{12-14}$
1,5,8,9 Department of Critical Care Medicine, Mahatma Gandhi Medical College and Hospital, Jaipur, Rajasthan, India

${ }^{2}$ Department of Respiratory Medicine, Mahatma Gandhi Medical College and Hospital, Jaipur, Rajasthan, India

${ }^{3}$ Department of General Medicine, Mahatma Gandhi Medical College and Hospital, Jaipur, Rajasthan, India

4,7Department of Medicine, Mahatma Gandhi Medical College and Hospital, Jaipur, Rajasthan, India

${ }^{6}$ Mahatma Gandhi Medical College and Hospital, Jaipur, Rajasthan, India

Corresponding Author: Srishti S Jain, Department of Critical Care Medicine, Mahatma Gandhi Medical College and Hospital, Jaipur, Rajasthan, India, Phone: +91 7820895064, e-mail: srishti5484@gmail. com

How to cite this article: Jain SS, Jain A, Kalia A, et al. Impact of Triaging for COVID-19 Patients in a Tertiary Care Hospital in West India: A Retrospective Analysis of a Case Series. J Mahatma Gandhi Univ Med Sci Tech 2021;6(1):5-9.

Source of support:

Conflict of interest:

There is wide variability in the clinical presentation and laboratory marker profiles of COVID-19 patients. In this descriptive

(0) The Author(s). 2021 Open Access This article is distributed under the terms of the Creative Commons Attribution 4.0 International License (https:// creativecommons.org/licenses/by-nc/4.0/), which permits unrestricted use, distribution, and non-commercial reproduction in any medium, provided you give appropriate credit to the original author(s) and the source, provide a link to the Creative Commons license, and indicate if changes were made. The Creative Commons Public Domain Dedication waiver (http://creativecommons.org/publicdomain/zero/1.0/) applies to the data made available in this article, unless otherwise stated. 
analysis of deceased patients at our institute, we hypothesized that mortality rates should be different according to the level of care offered at admission, as the patients triaged to ICU are considered vulnerable and their illness is at an advanced stage. ${ }^{3,5}$

\section{Materials and Methods}

It is a retrospective observational descriptive study of mortality data of patients admitted to a Tertiary Care Hospital in North India during the peak of pandemic in the month of September to December 2020. Patients were admitted and handled as per the contemporary interim guidance issued by the MOHFW of India during the pandemic. All the clinical data were collected retrospectively in the standard clinical research forms. This retrospective analytic study was approved by the Internal Ethics Committee and as there was no active involvement of human subjects in this study, a consent waiver was also given by the Institutional Ethics Committee (IEC).

All patients underwent an HRCT thorax, routine lab investigations, and point-of-care immunoanalysis which gives D-dimer, troponin T, procalcitonin, and NT-proBNP on admission. Baseline Charlson's comorbidity index was calculated for all these patients depending upon their comorbidities.

The admission value and the maximum value of these biomarkers were taken from the electronic medical record of the patients retrospectively.

\section{Data Analysis}

These data were prepared and an interim evaluation was done. During the preliminary analysis, we could find a few outliers in continuous series, hence median with IQR was used as appropriate, for descriptive statistics, and mean and the standard deviation is applied for normally distributed variables.

We further divided these observations into two cohorts according to the care they received at their admission and analyzed their first biomarker values and other variables for any statistical difference. The significance of other variables, the proportion of ward, and the ICU admission's fatal outcome were evaluated using Chi-square statistics, paired "T-test", and "Mann-Whitney $U$ test" as found suitable. A $p$ value of $<0.05$ was accepted as statistically significant. All the statistical analyses were performed using Xlstat software.

\section{Results}

This single-center case series describes the fatalities of SARS-CoV-2 confirmed cases from September 1 to December 31, 2020. There were a total of 2,689 SARS-CoV-2 cases admitted during this period, and the case distribution is displayed in Flowchart 1.

The patients were either admitted in the ward or the ICU as per the clinical case severity definitions. ${ }^{7}$ Patients with mild and moderate disease severity were admitted to the ward and all the severe cases were admitted to the ICU as per the discretion of the admitting physician.

Based on the above criteria, 1,849 (68.76\%) patients were initially admitted to the ward and 840 (31.24\%) patients were directly admitted to the ICU. From the ward, 1,740 patients were discharged and 109 patients died giving a percentage of $5.89 \%$ mortality. In ICU, 840 patients were admitted out of which 784 patients were discharged alive and 56 deaths were recorded. The percentage mortality from ICU was $6.67 \%$.
Flowchart 1: Flow diagram showing analyzed case

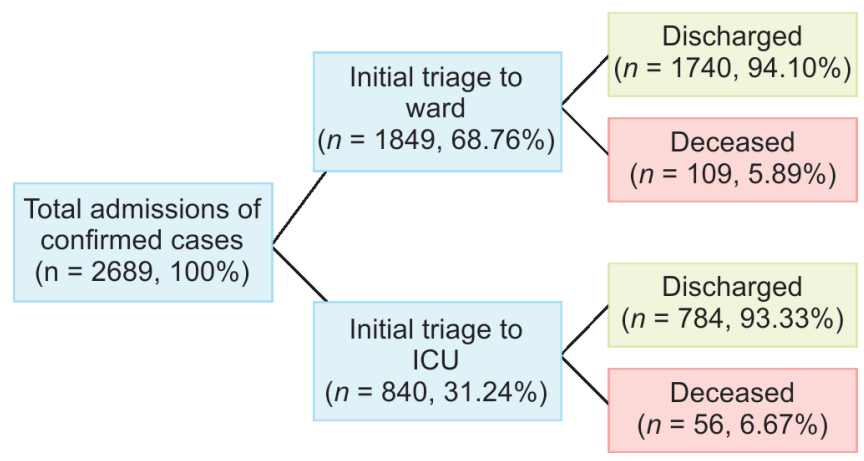

Demographic data and biomarker profiles of these patients are presented in Table 1.

The median (IQR) age in years of all the admissions was 67 (57.75-74) years. Males were affected almost 3 times more than females. The median (IQR) duration of admission and hospital stay was $7^{3-12}$ days (Table 1). Diabetes was the most common comorbidity along with hypertension among the COVID-affected dead patients (Table 2).

For the comparison and subgroup analysis, these patients were divided into two groups: those patients who were admitted in the ward on admission and those who were admitted in the ICU.

The median (IQR) D-dimer (in $\mu \mathrm{g} / \mathrm{L}$ ) of ward patients was 3,580 $(1,530-9,225)$ and that of the ICU was 4,025 $(1,995-18,275)$. Median (IQR) NT-proBNP (in ng/L) for patients admitted to wards was 2,600 (717.25-6,085) and that of ICU was 3,030 (877.5-10,200). The median (IQR) procalcitonin (PCT) (in $\mathrm{ng} / \mathrm{mL}$ ) of the patients admitted to the ward was $0.92(0.22-3)$ and $0.845(0.25-2.77)$ of the ICU. The median (IQR) D-dimer (in $\mu \mathrm{g} / \mathrm{L}$ ) of the patients admitted to the ward was $14,800(6,100-52,900)$, whereas it was $10,655(3,512.5-52,100)$ for the patients admitted to the ICU. Highest NT-proBNP (in $\mathrm{ng} / \mathrm{L}$ ) for patients admitted to the ward was $5,420(2,670-19,800)$ and was $9,660(3,115-18,850)$ for ICU patients. The median (IQR) of the highest troponin $\mathrm{T}$ (in $\mathrm{ng} / \mathrm{L}$ ) for patients admitted to the ward was 0.098 (0.037-0.36) and 0.14 (0.039-0.71) for ICU patients. The median (IQR) of the highest procalcitonin was $3.5(0.82-13.25)$ for patients in the ward and 3.8 (1.1-16) for ICU patients.

Statistical analysis of these data revealed that there was no significant difference among both the subgroups; however, one may postulate that the admission to ICU should carry higher odds of adverse overall outcomes considering the advanced stage of their disease (Fig. 1).

\section{Discussions}

This is a case series and retrospective analysis of fatal outcome data during the concluded wave of the pandemic of COVID-19. We included 165 fatal outcome patients and analyzed demographics and laboratory risk factor data after dividing them into two subgroups of ward vs ICU admission. We found that both the groups were comparable on all the evaluated parameters and had similar fatal outcome profiles despite having differences in the initial clinical severity.

In our study, we report that the majority of deaths were from the median 67 (57.75-74) years age-group and most of the deceased were males (74\%). Their average duration of stay was 7 days. And they had similar comorbidity profiles. After evaluating the cohorts, we could not find any difference among both groups. 
Table 1: Demographic and biomarker variables

\begin{tabular}{|c|c|c|c|c|}
\hline & All in-hospital mortalities & $\begin{array}{l}\text { All mortalities from } \\
\text { initial ward admission }\end{array}$ & $\begin{array}{l}\text { All mortalities from initial } \\
\text { ICU admission }\end{array}$ & pvalue \\
\hline Duration of stay (in days) median (IQR) & $7(3-12)$ & $7(3-13)$ & $6.5(410)$ & 0.82 \\
\hline Age (in years) median (IQR) & $67(57.75-74)$ & $68(59-74)$ & $62.5(54.5-74)$ & 0.18 \\
\hline Sex (M:F) (proportion) & $123: 42$ & $78: 31$ & $45: 11$ & 0.21 \\
\hline D-dimer (P) ( $\mu \mathrm{g} / \mathrm{L})$ on DOA median (IQR) & $3,580(1,622.5-10,375)$ & $3,580(1,530-9,225)$ & $4,025(1,995-18,275)$ & 0.41 \\
\hline NT-proBNP (ng/L) on DOA median (IQR) & $2,730(772-7,435)$ & $2,600(717.25-6,085)$ & $3,030(877.5-10,200)$ & 0.45 \\
\hline TnT (ng/mL) on DOA median (IQR) & $0.025(0.01-0.12)$ & $0.0205(0.01-0.11)$ & $0.046(0.01-0.14)$ & 0.42 \\
\hline PCT (ng/mL) on DOA median (IQR) & $0.91(0.225-2.65)$ & $0.92(0.22-3)$ & $0.845(0.2525-2.775)$ & 0.73 \\
\hline D-dimer (P) ( $\mu \mathrm{g} / \mathrm{L})$ highest median (IQR) & $13,200(5,330-50,400)$ & $14,800(6,100-52,900)$ & $10,655(3,512.5-52,100)$ & 0.35 \\
\hline NT-proBNP (ng/L) highest median (IQR) & $6,070(2,745-18,450)$ & $5,420(2,670-19,800)$ & $9,660(3,115-18,850)$ & 0.38 \\
\hline TnT (ng/mL) highest median (IQR) & $0.11(0.0375-0.43)$ & $0.098(0.037-0.36)$ & $0.14(0.039-0.71)$ & 0.57 \\
\hline PCT (ng/mL) highest median (IQR) & $3.7(0.9775-13.75)$ & $3.5(0.82-13.25)$ & $3.8(1.1-16)$ & 0.59 \\
\hline \multicolumn{5}{|l|}{ Charlson's comorbidity index } \\
\hline$<2$ & 41 & 27 & 14 & $\begin{array}{l}p \text { value } 0.975 \text { by } \\
\text { Wilk's } G \text { test }\end{array}$ \\
\hline $2-5$ & 116 & 77 & 39 & \\
\hline$>5$ & 8 & 5 & 3 & \\
\hline
\end{tabular}

ICU, intensive care unit; IQR, interquartile range; PCT, procalcitonin; TnT, Troponin T; $\mu$ g/L, microgram/liter; ng/mL, nanogram/milliliter; NT-proBNP, N terminal pro brain natriuretic peptide

Table 2: Comorbidities of all the patients

\begin{tabular}{llll}
\hline Comorbidity & Total $(n)$ & Ward & ICU \\
\hline Diabetes & 35 & 19 & 16 \\
Hypertension & 23 & 10 & 13 \\
Chronic kidney disease & 11 & 6 & 5 \\
$\begin{array}{l}\text { Coronary artery disease/myocardial } \\
\text { infarction }\end{array}$ & 5 & 2 & 3 \\
Malignancy & 3 & 1 & 2 \\
Hypothyroidism & 3 & 1 & 2 \\
$\begin{array}{l}\text { Chronic obstructive pulmonary dis- } \\
\text { ease/asthma }\end{array}$ & 3 & 0 & 3 \\
Cerebrovascular disease & 1 & & \\
\hline
\end{tabular}

An initial large epidemiological study from China that includes 72,314 patients during the epidemic reports that the severity of COVID-19 ranges from mild to severe. ${ }^{15}$ And approximately $81 \%$ of cases are classified in the mild to moderate category, $14 \%$ in the severe, and $5 \%$ in the critical category; however, their inclusion criteria to the moderate category also have a radiological feature ( $>50 \%$ lung involvement on imaging), this study reports that most affected population subgroups was from 30 to 79 years.

A previous study from Ireland estimates that 60.7 and $33.6 \%$ of admission to critical care units were from the ward and emergency rooms, respectively. They had a similar median age of 60 years and $70.1 \%$ male admission in ICUs during their study period. ${ }^{16}$

In another study published during the initial months of the pandemic from Mainland China suggests that among the nonsurvivors $75.9 \%$ of patients were from $>60$ years age-group and a majority $(55.6 \%)$ of them were male. ${ }^{17}$ This was one of the initial works where a strong linkage of raised biomarkers (CRP, D-dimer, PCT, LDH, ferritin, and IL- 6 were postulated). Another work from the USA suggests that there is significant age and sex difference among the non-survivors and survivors. ${ }^{18}$ They evaluated 2,215 adult patients data who were admitted to 55 ICUs. They report that approximately $35.4 \%$ of patients had died at the end of 28 days. The mean age was $66(13.3)$ years and $68.4 \%$ were male among the non-survivor group.

As already depicted in Table 1, our patients have higher laboratory biomarker levels at admission and maximum values during the admission, than the normal reference range and there was no significant difference available among the two selected cohorts in our study. A similar strong linkage between raised biomarkers and fatality outcome has already been established in many previous studies. ${ }^{9-11,16-20}$

Hence, the hypothesis that initial ICU admission and ward admission patients should not have a difference in mortality based on the disease severity at the time of triage, could not be rejected and we could conclude that apart from clinical screening for disease severity, a point-of-care testing for biomarker levels, and a radiology-based algorithm to ascertain the disease severity will be a rather robust predictor of disease severity, and final outcome.

\section{Strength and Limitations}

Our case series analysis tests a unique clinical question and provides a new dimension to explore in the COVID-19 management in a properly designed robust prospective clinical research protocol. However, due to a serious paucity of available data, we are presently not able to provide any concrete associations and correlation statistics. Due to different schemes of presentation of data in epidemiological and descriptive studies in COVID-19, we are not able to make suitable comparisons for initial triaging and outcomes also with our work. We assume that our work may help in the evolution of individual institutional protocols for triaging and management of COVID-19, and also acknowledge the fact that indiscriminate use of such protocols will further strain the already ailing medical capacity of the state.

\section{Conclusion}

Our retrospective analysis of mortality data suggests that despite adequate clinical screening, the initial assignment of level of care 

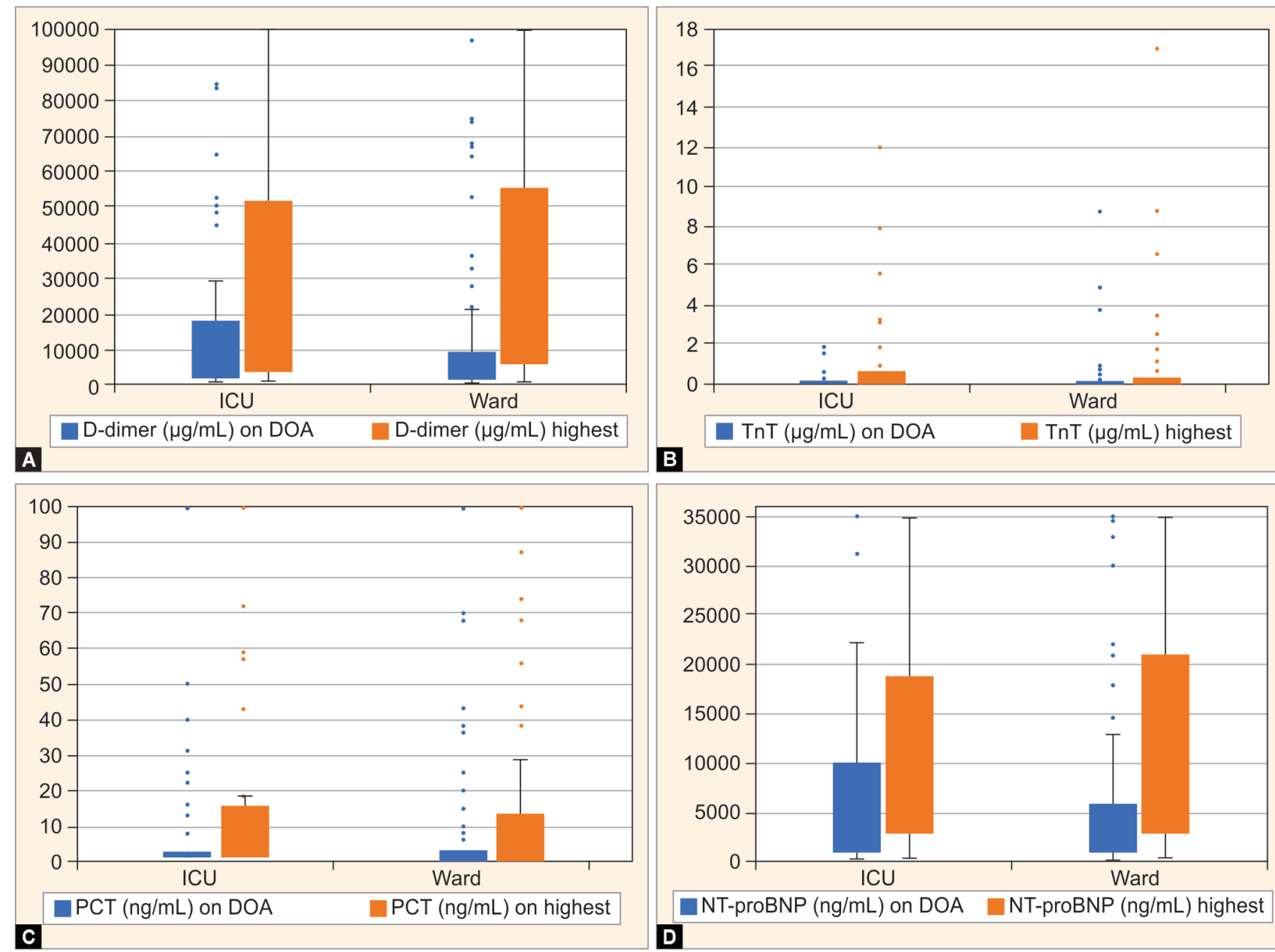

B

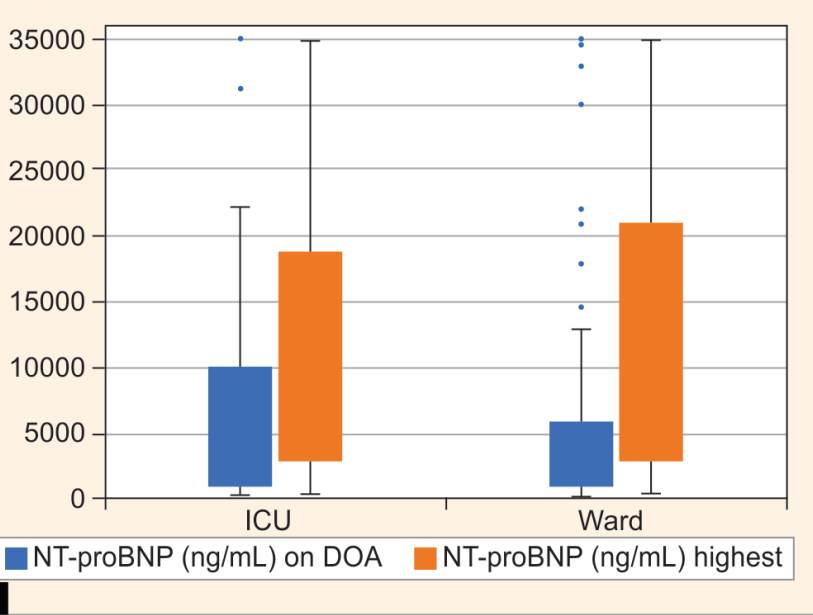

Figs 1 A to D: Comparison of biomarker values between ward and ICU fatalities: (A) D-dimer (Ward vs ICU); (B) TnT (Ward vs ICU); (C) PCT (Ward vs ICU); (D) NT-proBNP (Ward vs ICU)

at the time of admission failed to impose any impact on the final adverse outcome. Thus, we could conclude that clinical criteria for disease severity need suitable modifications and the addition of point-of-care biomarker testing or radiology-based triaging algorithms would be appropriate before the next wave of pandemic sets in the region.

The very concept of this work raises an important issue of adequate triaging of COVID-19 patients and survival. Nine international guidelines for triaging ICU beds during the pandemic of COVID-19 were reviewed systematically. ${ }^{21}$ These guidelines covered a common theme of ethical framework, criteria for ICU admission and discharge, adaptation during changing demand, equitable and judicious distribution of resources. The reviewer provides insight that there are vast differences among these guidelines even in similar regions of the world; hence, our proposed modification will also be tested against their feasibility of mass implication in the event of the surge and straining medical facilities in the state.

\section{References}

1. WHO Coronavirus Disease (COVID-19) Dashboard [Internet]. [cited 2021 Mar 1]. Available from: https://covid19.who.int.

2. Macera M, De Angelis G, Sagnelli C, et al. Clinical presentation of COVID-19: case series and review of the literature. Int J Environ Res Public Health 2020;17(14):5062. DOI: 10.3390/ijerph17145062.
Available from: https://www.ncbi.nlm.nih.gov/pmc/articles/ PMC7399865/.

3. Wang D, Hu B, Hu C, et al. Clinical characteristics of 138 hospitalized patients with 2019 novel coronavirus-infected pneumonia in Wuhan, China. JAMA 2020;323(11):1061. DOI: 10.1001/jama.2020.1585. Available from: https://jamanetwork.com/journals/jama/fullarticle/ 2761044.

4. XuX-W, Wu X-X, Jiang X-G, et al. Clinical findings in a group of patients infected with the 2019 novel coronavirus (SARS-Cov-2) outside of Wuhan, China: retrospective case series. BMJ 2020. m606. Available from: https://www.bmj.com/lookup/doi/10.1136/bmj.m606.

5. Yang $X, Y u ~ Y, X u ~ J$, et al. Clinical course and outcomes of critically ill patients with SARS-CoV-2 pneumonia in Wuhan, China: a single-centered, retrospective, observational study. Lancet Respir Med 2020;8(5):475-481. DOI: 10.1016/S2213-2600(20)30079-5. Available from: https://linkinghub.elsevier.com/retrieve/pii/ S2213260020300795.

6. Zhou F, Yu T, Du R, et al. Clinical course and risk factors for mortality of adult inpatients with COVID-19 in Wuhan, China: a retrospective cohort study. Lancet Lond Engl. 2020;395(10229):1054-1062. DOI: 10.1016/S0140-6736(20)30566-3.

7. UpdatedClinicalManagementProtocolforCOVID19dated03072020. pdf [Internet]. [cited 2021 Mar 1]. Available from: https:// www.mohfw.gov.in/pdf/UpdatedClinicalManagement ProtocolforCOVID19dated03072020.pdf.

8. Clinical Spectrum [Internet]. COVID-19 Treatment Guidelines. [cited 2021 Mar 1]. Available from: https://www.covid19treatmentguidelines. nih.gov/overview/clinical-spectrum/. 
9. Zhang L, Yan X, Fan Q, et al. D-dimer levels on admission to predict in-hospital mortality in patients with Covid-19. J Thromb Haemost JTH 2020;18(6):1324-1329. DOI: 10.1111/jth.14859.

10. Chen H, Li X, Marmar T, et al. Cardiac troponin I association with critical illness and death risk in 726 seriously ill COVID-19 patients: a retrospective cohort study. Int J Med Sci 2021;18(6):1474-1483. DOI: 10.7150/ijms.53641.

11. Tan C, Huang Y, Shi F, et al. C-reactive protein correlates with computed tomographic findings and predicts severe COVID-19 early. J Med Virol 2020;92(7):856-862. DOI: 10.1002/jmv.25871.

12. Investigation of novel SARS-CoV-2 variant - Variant of Concern 202012/01. [cited 2021 Mar 1];19. Available from: https://www.gov.uk/ government/publications/investigation-of-novel-sars-cov-2-variantvariant-of-concern-20201201.

13. Wise J. COVID-19: new coronavirus variant is identified in UK. BMJ 2020;371:m4857. DOI: 10.1136/bmj.m4857. Available from: https:// www.bmj.com/content/371/bmj.m4857.

14. Risk of spread of new SARS-CoV-2 variants of concern in the EU/EEA - first update. 2021; 29. Available from https://www.ecdc.europa. eu/en/publications-data/covid-19-risk-assessment-spread-newvariants-concern-eueea-first-update.

15. Wu Z, McGoogan JM. Characteristics of and important lessons from the coronavirus disease 2019 (COVID-19) outbreak in China: summary of a report of 72314 cases from the Chinese Center for Disease Control and Prevention. JAMA 2020;323(13):1239-1242. DOI: 10.1001/ jama.2020.2648.
16. Richards-Belle A, Orzechowska I, Gould DW, et al. COVID-19 in critical care: epidemiology of the first epidemic wave across England, Wales and Northern Ireland. Intensive Care Med 2020;46(11):2035-2047. Available from: 10.1007/s00134-020-06267-0.

17. Wang Y, Lu X, Li Y, et al. Clinical course and outcomes of 344 intensive care patients with COVID-19. Am J Respir Crit Care Med 2020;201(11):1430-1434. Available from: https://www.atsjournals. org/doi/10.1164/rccm.202003-0736LE.

18. Gupta S, Hayek SS, Wang W, et al. Factors associated with death in critically III patients with coronavirus disease 2019 in the US. JAMA Intern Med 2020;180(11):1436. DOI: 10.1001/jamainternme d.2020.3596Available from: https://jamanetwork.com/journals/ jamainternalmedicine/fullarticle/2768602.

19. Abdeen $Y$, Kaako A, Alnabulsi $M$, et al. The prognostic effect of brain natriuretic peptide levels on outcomes of hospitalized patients with COVID-19. Avicenna J Med 2021;11(1):20-26. DOI: 10.4103/ajm. ajm_169_20.

20. Berger JS, Kunichoff D, Adhikari S, et al. Prevalence and outcomes of D-Dimer elevation in hospitalized patients with COVID-19. Arterioscler Thromb Vasc Biol 2020;40(10):2539-2547. DOI: 10.1161/ ATVBAHA.120.314872.

21. Tyrrell CSB, Mytton OT, Gentry SV, et al. Managing intensive care admissions when there are not enough beds during the COVID-19 pandemic: a systematic review. Thorax 2021;76(3):302-312. DOI: 10.1136/thoraxjnl-2020-215518. Available from: https://thorax.bmj. com/content/76/3/302. 